\title{
Seasonal variation in occurrence of aortic diseases: The database of hospital discharge data of the Emilia-Romagna region, Italy
}

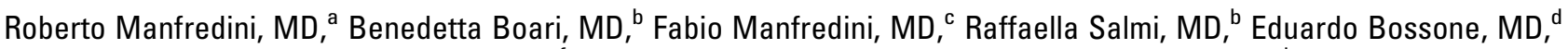 \\ Davide Fabbri, MD, ${ }^{\mathrm{e}}$ Edgardo Contato, MD, ${ }^{f}$ Francesco Mascoli, MD, ${ }^{\mathrm{g}}$ and Massimo Gallerani, MD, ${ }^{\mathrm{b}}$ Ferrara and Milan, Italy
}

$\mathrm{I}$ $\mathrm{n}$ analogy with other cardiovascular events, such as myocardial infarction and stroke, acute aortic diseases do not randomly occur over time but show definite chronobiologic variations characterized by a higher frequency during morning hours and winter months. ${ }^{1-3}$ This study was aimed to confirm, based on the large number of cases in a regional database, the existence of a seasonal variation in acute aortic diseases.

\section{Clinical Summary}

We used the database of all hospital admissions of the Emilia-Romagna region, Italy, Center for Health Statistics, between January 2000 and December 2006. Emilia-Romagna is situated in northeastern Italy, extending from the Po River to the north, the Adriatic Sea to the east, and the Appennini mountains to the south and west. It has a surface of $22,124 \mathrm{~km}^{2}$ and a population of approximately $3,985,000$ persons ( $7 \%$ of the Italian population). The close proximity to the Adriatic Sea does not exert significant influence on the climate, which is continental and characterized by significant temperature digression from the different seasons, with hot summers and rainy autumns. The database contains all discharge hospital sheets of the patients admitted to all area public and private hospitals, a total of approximately 6 million admissions (latest database update, December 2006). The discharge hospital sheet contains each patient's demographic data, date and department of admission and discharge, and up to 9 discharge diagnoses coded according to the International Classification of Diseases, 9th Revision, Clinical Modification (ICD-9-CM). We considered all cases with the follow-

From the Department of Internal Medicine, ${ }^{a}$ Hospital of the Delta, Azienda Unità Sanitaria Locale di Ferrara, and the Department of Clinical and Experimental Medicine, ${ }^{a}$ Section of Clinica Medica, and the Vascular Diseases Center, University of Ferrara, Italy; the Department of Internal Medicine, ${ }^{\text {b }}$ Azienda Ospedaliera-Universitaria S. Anna, Ferrara, Italy; the Vascular Rehabilitation Program, ${ }^{\mathrm{c}}$ Vascular Diseases Center, University of Ferrara, Italy; the Cardiology Division, ${ }^{\mathrm{d}}$ Cava dè Tirreni and Amalfi Coast Hospital, Salerno, Italy, and the Cardiorespiratory Department, ${ }^{\mathrm{d}}$ University of Milan, Milan, Italy; Medical Direction, ${ }^{\mathrm{e}}$ Azienda OspedalieraUniversitaria S. Anna, Ferrara, Italy; Medical Direction, ${ }^{\mathrm{f}}$ Azienda Unità Sanitaria Locale, Ferrara, Italy; and the Department of Vascular Surgery, ${ }^{\mathrm{g}}$ Hospital of Ferrara, and the Vascular Diseases Center, University of Ferrara, Ferrara, Italy.

Received for publication Aug 22, 2007; accepted for publication Oct 19, 2007.

Address for reprints: Roberto Manfredini, MD, Department of Clinical and Experimental Medicine, Section of Clinica Medica and Vascular Diseases Center, University of Ferrara, via Savonarola 9, 44100 Ferrara, Italy (E-mail:mfr@unife.it).

J Thorac Cardiovasc Surg 2008;135:442-4

$0022-5223 / \$ 34.00$

Copyright (C) 2008 by The American Association for Thoracic Surgery doi:10.1016/j.jtcvs.2007.10.032
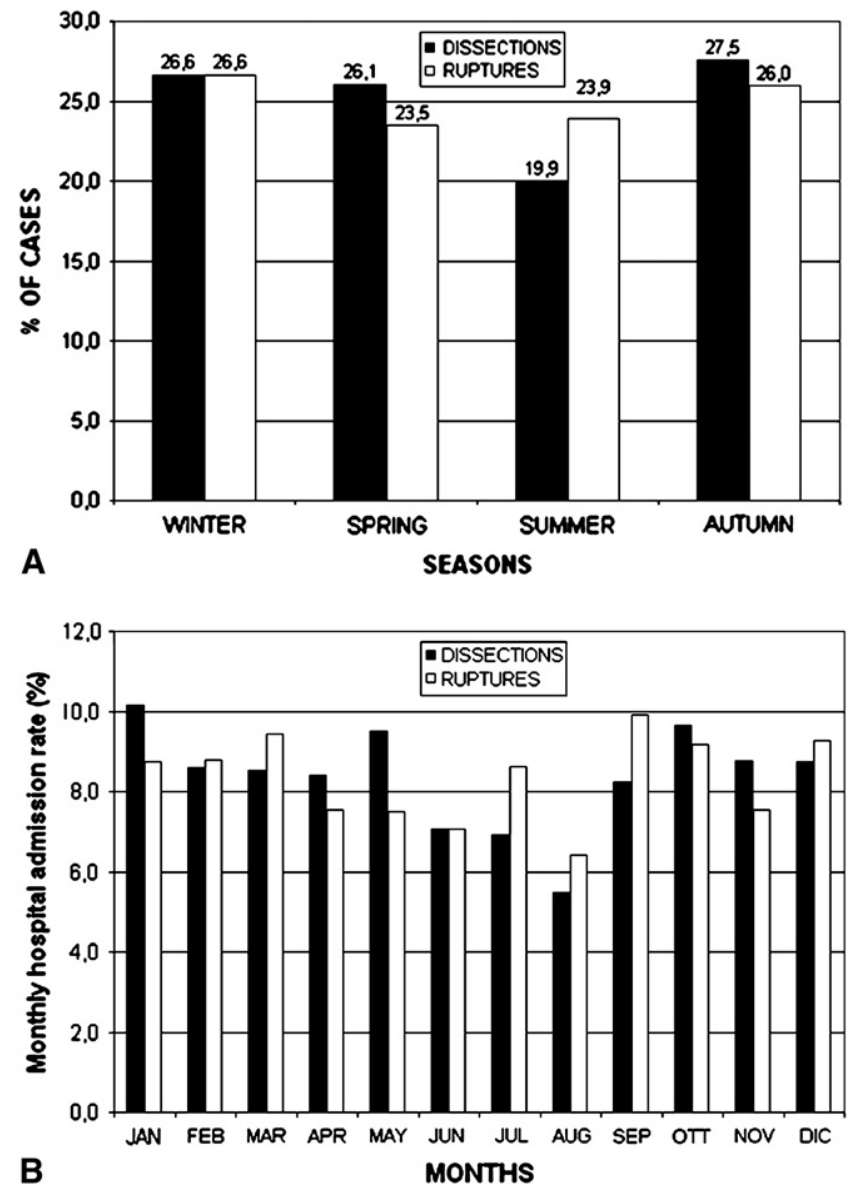

Figure 1. Acute aortic diseases: seasonal distribution of percentage of cases $(A)$ and monthly distribution of hospital admission rate (B).

ing ICD-9-CM codes: 441, aortic aneurysm and dissection (excluding syphilitic aortic aneurysm [093.0] and traumatic aortic aneurysm [901.0 and 902.0]); 441.0, dissection of aorta; 441.00, unspecified site; 441.01, thoracic; 441.02, abdominal; 441.03, thoracoabdominal; 441.1, thoracic aneurysm, ruptured; 441.3, abdominal aneurysm, ruptured; 441.5, aortic aneurysm of unspecified site; and 441.6, thoracoabdominal aneurysm, ruptured.

Cases were categorized by month of onset of each event, and the $\chi^{2}$ test for goodness of fit was used for statistical analysis on the total population and subgroups according to sex, age, and type of acute event, such as dissection or rupture.

The total sample consisted of 4615 subjects (77\% male subjects; mean age, $70 \pm 13$ years; $59.8 \%$ dissections and $40.2 \%$ ruptures). 
TABLE 1. Seasonal distribution of acute aortic diseases

\begin{tabular}{|c|c|c|c|c|c|c|}
\hline & Winter, n (\%) & Spring, n (\%) & Summer, n (\%) & Autumn, n (\%) & $\chi^{2}$ & $P$ value \\
\hline \multicolumn{7}{|l|}{ Total } \\
\hline All & $1227(26.6)$ & $1155(25.0)$ & $991(21.5)$ & $1242(26.9)$ & 34.38 & $<.0001$ \\
\hline Male subjects & $890(26.4)$ & $831(24.6)$ & $728(21.6)$ & $923(27.4)$ & 26.07 & $<.0001$ \\
\hline Female subjects & $337(27.1)$ & $324(26.1)$ & $263(21.2)$ & $319(25.7)$ & 10.37 & .017 \\
\hline$<60 \mathrm{y}$ & $234(27.9)$ & $210(25.0)$ & $149(17.8)$ & $246(29.3)$ & 26.77 & $<.0001$ \\
\hline $61-74$ y & $497(26.8)$ & 481 (25.9) & $402(21.7)$ & $475(25.6)$ & 11.54 & .01 \\
\hline$>75 y$ & $496(25.8)$ & $464(24.2)$ & $440(22.9)$ & $521(27.1)$ & 7.9 & .049 \\
\hline \multicolumn{7}{|l|}{ Dissections } \\
\hline All* & $733(26.6)$ & $719(26.1)$ & $548(19.9)$ & $760(27.5)$ & 40.22 & $<.0001$ \\
\hline Thoracic aorta & $357(27.9)$ & $318(24.8)$ & $255(19.9)$ & $350(27.3)$ & 20.31 & $<.0001$ \\
\hline Abdominal aorta & $145(26.3)$ & $160(29.0)$ & $107(19.4)$ & $140(25.4)$ & 10.86 & .013 \\
\hline $\begin{array}{l}\text { Thoracoabdominal } \\
\text { aorta }\end{array}$ & $164(23.8)$ & $187(27.1)$ & $133(19.3)$ & $206(29.9)$ & 17.24 & .001 \\
\hline Unspecified site & $67(28.2)$ & $54(22.7)$ & $53(22.3)$ & $64(26.9)$ & 2.54 & .475 \\
\hline \multicolumn{7}{|l|}{ Ruptures } \\
\hline All $\dagger$ & $494(26.6)$ & $436(23.5)$ & $443(23.9)$ & $482(26.0)$ & 5.29 & .154 \\
\hline Thoracic aorta & $81(28.0)$ & $67(23.2)$ & $64(22.1)$ & $77(26.6)$ & 2.71 & .442 \\
\hline Abdominal aorta & $367(25.9)$ & $337(23.8)$ & $346(24.4)$ & $368(26.0)$ & 2.03 & .568 \\
\hline $\begin{array}{l}\text { Thoracoabdominal } \\
\text { aorta }\end{array}$ & $46(31.1)$ & $32(21.6)$ & $33(22.3)$ & $37(25.0)$ & 3.3 & .349 \\
\hline \multicolumn{7}{|l|}{ Hypertensive subjects } \\
\hline All & $357(26.5)$ & $349(25.9)$ & $288(21.3)$ & $355(26.3)$ & 9.7 & .022 \\
\hline Dissection & $246(26.2)$ & $259(27.6)$ & $177(18.9)$ & $256(27.3)$ & 19.24 & $<.0001$ \\
\hline Rupture & $111(27.0)$ & $90(21.9)$ & $111(27.0)$ & $99(24.1)$ & 3.09 & .386 \\
\hline \multicolumn{7}{|l|}{ Normotensive subjects } \\
\hline All & $870(26.6)$ & $806(24.7)$ & $703(21.5)$ & $887(27.2)$ & 25.52 & $<.0001$ \\
\hline Dissection & $487(26.7)$ & $460(25.2)$ & $371(20.4)$ & $504(27.7)$ & 23.09 & $<.0001$ \\
\hline Rupture & $383(26.5)$ & $346(24.0)$ & $332(23.0)$ & $383(26.5)$ & 5.63 & .132 \\
\hline
\end{tabular}

Mean age: ${ }^{*} 66.7 \pm 13.6$ years vs $\dagger 75.3 \pm 10.5$ years; $\mathrm{t}=22.210 ; P<.0001$.

Hypertension was present in 34\% of patients with dissection and in $22.2 \%$ of patients with rupture, respectively. A seasonal pattern characterized by lowest frequency in summer was shown for total cases $\left(\chi^{2}=34.38, P<.0001\right)$ and subgroups by sex and age (Table 1). Separate analysis by type of event confirmed this seasonal pattern for dissection but not for the rupture subgroup $\left(\chi^{2}=40.1[P\right.$ $<.0001$ ] and $\chi^{2}=4.96$ [not significant], respectively) independently of the presence of hypertension (Table 1 and Figure 1, A). The reduced frequency of events during the summer was confirmed also by using the monthly admission rate analysis (ie, the total number of events per each month of the year divided by the total number of hospital admissions of all kinds per each of the corresponding months for the entire study period; Figure 1, $B$ ).

\section{Discussion}

Previous studies on the seasonal variation in the occurrence of aortic diseases identified an increased frequency in winter. ${ }^{3}$ The main finding of this study is the observation of a significant summer low rather than a winter high. Interestingly, the lower frequency rate of aortic dissections in summer $(19.9 \%)$ is quite similar to that (19.4\%) found in the worldwide population of the International Registry of Acute Aortic Dissection. ${ }^{4}$ It is likely that summer months might have a protective effect against all those factors related to cold exposure, such as increased blood pressure and arterial spasm, platelet and red blood cell count, blood viscosity, lipid levels, and clotting activity, known to be important risk factors for the winter preference of acute aortic and cardiovascular events. ${ }^{4}$ Summer, in fact, has been associated with the lowest and winter with the highest clinic blood pressure in both normal and increased blood pressure values, regardless of the effect of antihypertensive drugs. ${ }^{5}$ The difference of patterns between dissection and rupture might reflect the pathophysiologic differences between these 2 clinical entities, where the increase in sympathetic activity and blood pressure plays a major role, especially for dissection.

On one hand, this study conducted on discharge ICD-9-CM data has a main limitation in the accuracy of the coding process itself. On the other hand, however, it allows us to examine a large number of cases not easily available in the literature. Further studies aimed to prospectively investigate the relationships between meteorologic, pathophysiologic, and hematochemical factors are needed.

\section{References}

1. Gallerani M, Portaluppi F, Grandi E, Manfredini R. Circadian rhythmicity in the occurrence of spontaneous acute dissection and rupture of thoracic aorta. J Thorac Cardiovasc Surg. 1997;113:603-4.

2. Manfredini R, Portaluppi F, Zamboni P, Salmi R, Gallerani M. Circadian variation in spontaneous rupture of abdominal aorta. Lancet. 1999;353: 643-4.

3. Manfredini R, Boari B, Gallerani M, Salmi R, Bossone E, Distante A, et al. Chronobiology of rupture and dissection of aortic aneurysms. J Vasc Surg. 2004;40:382-8. 
4. Mehta RH, Manfredini R, Hassan F, Sechtem U, Bossone E, Oh JK, et al. Chronobiological patterns of acute aortic dissections. Circulation. 2002; 106:1110-5.
5. Sega R, Cesana G, Bombelli M, Grassi G, Stella ML, Zanchetti A, et al. Seasonal variations in home and ambulatory blood pressure in the PAMELA population. Pressione Arteriose Monitorate e Loro Associazioni. J Hypertens. 1998;16:1585-92.

\title{
Multiple thoracic aortic aneurysms after mediastinitis in an infant after repair of coarctation of the aorta
}

\author{
Laurie E. Profitlich, MD, ${ }^{*}$ Constance G. Weismann, MD, ${ }^{*}$ Shubika Srivastava, MD, Bruce D. Gelb, MD, \\ Kahn Nguyen, MD, and Umesh Joashi, MD, New York, NY
}

Supplemental material is available online.

\section{$\mathrm{W}$}

Clinical Summary e describe the case of a 3.1-kg term infant with critical aortic coarctation transferred to our institution on the second day after birth. There was no risk factor for infection in the perinatal or birth history. An umbilical arterial catheter (UAC) and venous catheters were placed and a prostaglandin $\mathrm{E}_{1}$ infusion was initiated. Intermittent low-grade fever on days 4 and 5 after birth was attributed to the prostaglandin therapy.

On day 6 , the patient was taken to the operating room for a resection and end-to-end anastomosis of a discrete coarctation through a left lateral thoracotomy. On entering the chest, we encountered purulent fluid in the mediastinum, and the aorta was adherent to the surrounding tissue, precluding mobilization. A subclavian flap repair was performed. The mediastinum was cultured and irrigated. The patient was transferred to the cardiac intensive care unit where vancomycin, cefepime, and gentamicin were started empirically. The UAC was removed. Osteomyelitis then developed. Blood and wound cultures persistently grew multi-drug resistant Staphylococcus aureus. The cultures finally became sterile on postoperative day 9 after modification of antibiotics (daptomycin, rifampin, and linezolid).

A chest computed tomogram (CT) performed on postoperative day 8 (Figure 1, A) showed multiple lung abscesses and a large, ring-enhancing mycotic aneurysm of the descending thoracic aorta

From the Department of Pediatric Cardiology, Mount Sinai Medical Center, New York, NY.

* Drs Profitlich and Weismann are joint first authors.

Received for publication Sept 7, 2007; accepted for publication Sept 20, 2007.

Address for reprints: Constance Weismann, MD, One Gustave L. Levy Place, New York, NY, 10029. (E-mail: constance.weismann@mssm.edu and laurie. profitlich@mssm.edu).

J Thorac Cardiovasc Surg 2008;135:444-5

$0022-5223 / \$ 34.00$

Copyright $\odot 2008$ by The American Association for Thoracic Surgery doi:10.1016/j.jtcvs.2007.09.024 measuring $1.1 \times 1.2 \mathrm{~cm}$ in continuity with a left-sided empyema. The nonaneurysmal aorta measured $0.6 \mathrm{~cm}$ in diameter. Despite antibiotic therapy and careful blood pressure control, a repeat chest CT done on postoperative day 13 (Figure $1, B$ ) showed a significant increase in the size of the aneurysm to $2.4 \times 1.5 \mathrm{~cm}$. Interval echocardiograms confirmed aneurysmal enlargement (Figure 2, $A$ and $B$ ). Also, a proximal aneurysm was detected just distal to the anastomosis of the subclavian flap.

A chest CT on postoperative day 28 (Figure 1, C) measured the distal aneurysm at $2.3 \times 3.9 \times 2.8 \mathrm{~cm}$ and the proximal aneurysm at $1.4 \times 2.2 \times 1.8 \mathrm{~cm}$. Owing to the rapidly increasing size and risk of rupture, an aortic bypass graft with exclusion of the entire thoracic descending aorta was performed on postoperative day 30. Via a median sternotomy, an 8-mm Hemashield graft (Boston Scientific, Natick, Mass) was anastomosed in an end-to-side fashion to the ascending aorta and to the descending aorta superior to the celiac trunk.

Eight days later, the patient hemorrhaged acutely from the mediastinal chest tube. A repeat chest CT (Figure 1,D) showed possible retrograde filling of the distal aortic aneurysm from collaterals supplied by intercostal arteries. Subsequent cardiac catheterization and angiography showed a widely patent aortic graft and confirmed retrograde filling of the aneurysm (Figure E1, $A$ and $B$ ).

A repeat chest CT 17 days after graft placement demonstrated continued filling of the thoracic aneurysm via intercostal arteries. The patient then underwent debulking of the aneurysm with removal of a large organized thrombus at that time. An actively bleeding intercostal artery was oversewn.

The patient recovered from the procedure well and was discharged home 8 days later receiving oral linezolid. At most recent follow-up, the patient was 8 months old and was enjoying normal growth and development. The graft was without anastomotic obstruction according to an echocardiographic assessment. Assuming normal somatic growth, we anticipate further surgical intervention will be necessary at the age of 5 or 6 years.

\section{Discussion}

Development of multiple aortic aneurysms in neonates associated with UAC placement is widely described in the literature. ${ }^{1}$ However, aortic aneurysm occurring secondary to mediastinal infection is rare.

UACs are placed in $10.8 \%$ to $64.4 \%$ of neonatal intensive care unit admissions. The incidence of complications is directly related to the duration of catheter use, ${ }^{2}$ and infection is strongly associated 